\title{
Pion-nucleon scattering in the cloudy bag model
}

\author{
E. A. Veit \\ Departamento de Fisica, Universidade Federal do Rio Grande do Sul, Rua Luiz Englert, S/N 90000, \\ Porto Alegre, Brasil \\ B. K. Jennings \\ TRIUMF, 4004 Wesbrook Mall, Vancouver, British Columbia, Canada V6T 2A3
}

A. W. Thomas

Department of Physics, The University of Adelaide, G.P.O. Box 498, Adelaide, South Australia 5001, Australia

(Received 20 May 1985)

\begin{abstract}
We investigate $s$ - and $p$-wave pion-nucleon scattering in the volume-coupling version of the cloudy bag model. The earlier conclusions about the nature of the $\Delta(1232)$, based on surface coupling, are not altered. This model also provides a good description of the "small" $p$-wave phase shifts, and the $s$-wave scattering lengths. However, there are indications from the $s$ waves at energies above $100 \mathrm{MeV}$, and from the $P_{11}$ channel, that some physics is still missing.
\end{abstract}

\section{INTRODUCTION}

In recent years considerable progress has been made towards understanding the properties of low-lying baryons within the so-called chiral bag models. ${ }^{1-10}$ One of the first problems investigated in the cloudy bag model (CBM) was pion-nucleon scattering and, in particular, the nature of the $P_{33}$ resonance. ${ }^{4,11}$ Since then there have also been studies of the Roper resonance (in the $P_{11}$ channel ${ }^{12}$ ) as well as the low-energy behavior of the other $p$ waves. ${ }^{13}$

Initially the CBM was incapable of dealing with $s$-wave pion-nucleon scattering at all. ${ }^{14}$ However, after transforming to the version where the pion couples throughout the bag volume $e^{9,10}$ one automatically obtains a term (reminiscent of $\rho$-meson exchange) which does scatter in $s$ wave. In the Born approximation this term reproduces the Weinberg-Tomozawa result for the $s$-wave scattering lengths exactly.

In this paper we investigate the consequences of the volume-coupling version of the CBM for both $s$ - and $p$ wave pion-nucleon scattering from threshold to a pion laboratory energy of about $500 \mathrm{MeV}$.

In order to put this work in context one should realize that the structure of the nucleon is still a very controversial topic. Many issues like the size of the quark core and the importance of (possibly) nonlinear mesonic effects are still being hotly debated. For this reason alone it is important that each model be tested against as many different experiments as possible. In this regard the recent work on pion production ${ }^{15}(\pi N \rightarrow \pi \pi N)$ and pion photoproduction (where the volume-type, derivative coupling was essential) ${ }^{16}$ has been extremely important.

The present investigations are a natural continuation of work begun on the $\bar{K} N$ (Ref. 17) and $K N$ (Ref. 18) systems. Thus the formalism used here has already been explained in detail in Refs. 17 and 18. Here we merely review the essentials. In Sec. III we present the results of the calculations, and discuss them at length. Section IV contains concluding remarks and some speculation on future developments.

\section{FORMALISM}

\section{A. The Lagrangian}

To order $\phi^{2}$ the Lagrangian density of the CBM [in its volume-coupling form for $\mathbf{S U}(2) \times \mathbf{S U}(2)]$ is written ${ }^{7,9,10,16}$

$$
\begin{aligned}
L(x)= & (i \bar{q} \partial q-B) \theta_{v}-\frac{1}{2} \bar{q} q \delta_{s}+\frac{1}{2}\left(\partial_{\mu} \vec{\phi}\right)^{2}-\frac{1}{2} m_{\pi}^{2} \vec{\phi}^{2} \\
& +\frac{\theta_{v}}{2 f} \bar{q} \gamma^{\mu} \gamma_{5} \vec{\tau} q \cdot \partial_{\mu} \vec{\phi}-\frac{\theta_{v}}{(2 f)^{2}} \bar{q} \gamma^{\mu} \vec{\tau} q \cdot\left(\vec{\phi} \times \partial_{\mu} \vec{\phi}\right)
\end{aligned}
$$

As usual $q$ and $\phi$ are the quark and pion fields, $B$ the bag constant, $f=93 \mathrm{MeV}$ (the pion-decay constant), $V$ the bag volume, and $S$ its surface. In the present model it is crucial that the pion field be allowed inside the bag in order to have the last term in Eq. (2.1). While there have been strong objections to this aspect of the CBM it is not at all inconsistent with (for example) the ideas of Shuryak $^{19}$ - see Ref. 20 for a longer discussion. From the purely phenomenological point of view, however, it seems worthwhile to allow for the possibility that the pion may have a different mass inside the bag than in free space. ${ }^{21}$ This would mean an extra (chiral-symmetry-breaking) term in the Lagrangian density

$$
L_{\delta m}=-\frac{1}{2}(\delta m)^{2} \vec{\phi}^{2} \theta_{v} .
$$

With this extra term the pion has an effective mass inside the bag given by

$$
M_{\text {inside }}=\left[m_{\pi}{ }^{2}+(\delta m)^{2}\right]^{1 / 2} .
$$

Clearly the limit where $\delta m$ is very large would effec- 


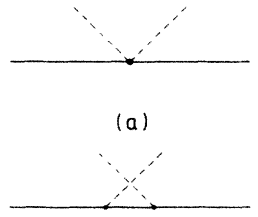

(c)

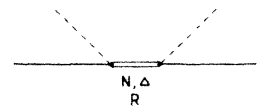

(b)

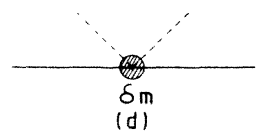

(d)
FIG. 1. Graphical representation of the four pieces of the effective meson-baryon potential used in this work: (a) contact interaction, (b) direct $s$-channel pole, (c) cross Born term, (d) phenomenological repulsion inside the bag (irrelevant except for $s$ waves).

tively exclude the pion from the bag interior.

We show in Fig. 1 the four distinct terms which contribute to pion-nucleon elastic scattering in lowest order. To calculate the $\pi N$ phase shifts we follow the usual CBM prescription $^{7,11}$ and solve a relativistic LippmannSchwinger equation, using these lowest-order diagrams as the driving potential. In the present case we allow both $\pi N$ and $\pi \Delta$ states and hence solve a coupled-channel problem. The use of the Lippman-Schwinger equation helps preserve the important property of unitarity which would be lost if we just calculated the $T$ matrix perturbatively.

The term shown in Fig. 1(a), which we call the contact term, is denoted $v_{\alpha \beta}^{c}$ where

$$
v_{\alpha \beta}^{c}=\left\langle\alpha\left|\int d^{3} x \frac{\theta_{v}}{(2 f)^{2}} \bar{q} \gamma^{\mu} \vec{\tau} q \cdot\left(\vec{\phi} \times \partial_{\mu} \vec{\phi}\right)\right| B\right\rangle .
$$

Here $\alpha$ and $\beta$ represent any pion-baryon channels-in the present case $\pi N$ or $\pi \Delta$. For the baryon states we use quark wave functions calculated in the MIT bag model. While this considerably simplifies our calculations, it does mean that important renormalization corrections, implicit in the CBM, ${ }^{7,11,17}$ are neglected. Thus we should not be too upset if the coupling constants needed to fit data differ by, say, (10-20)\% from naive expectations.

The preceding paragraph does not imply that the Lagrangian given in Eq. (2.1) is renormalizable. An important physical effect has been left out of Eq. (2.1): namely, the finite size of the pion. This would induce form factors and make the loops finite as occurs in the mesonexchange description of the nucleon-nucleon force. In the present case we have not included explicitly the pion form factor but have restricted the intermediate baryon states to be either nucleons or $\Delta$ 's. This assumes that the main role of the intrinsic pion size in the present calculation is suppressing the contribution of higher baryon resonances. The finite bag size generates form factors at the $\pi N N$, $\pi N \Delta$, etc., vertices which keep the diagrams we consider and those referred to in the last paragraph finite. Thus, at least in principle, the effects of these diagrams can be calculated explicitly.

Using a plane-wave basis for the mesons the time piece $(\mu=0)$ of Eq. (2.4) can be written ${ }^{17}$

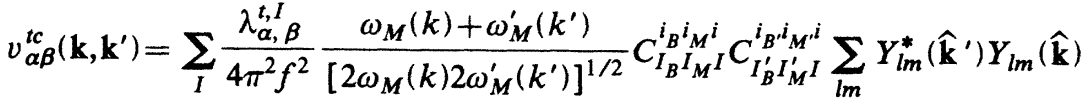

$$
\begin{aligned}
& \times N_{s}^{2} \int_{0}^{R} d x x^{2}\left[j_{0}^{2}\left(\omega_{s} x\right)+j_{1}^{2}\left(\omega_{s} x\right)\right] j_{1}(k x) j_{1}\left(k^{\prime}, x\right) .
\end{aligned}
$$

The coupling constant for the $\alpha \beta$ transition with total isospin $I$, labeled $\lambda_{\alpha \beta}^{t, I}$, is given in Table $I$. The isospin of the baryon (meson) is denoted $I_{B}\left(I_{M}\right)$, and its third component $i_{B}\left(i_{M}\right)$.

The spatial piece of the contact interaction yields the potential ${ }^{17}$

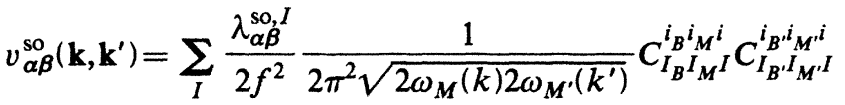

$$
\begin{aligned}
& \times \sum_{J M} \sum_{l, m, m^{\prime}} C_{l S_{B} J}^{m s_{B} M} C_{l S_{B^{\prime}} m^{\prime} s^{\prime}{ }^{M}} Y_{l m^{\prime}}^{*}\left(\hat{\mathbf{k}}^{\prime}\right) Y_{l m}(\hat{\mathbf{k}})(-2) \sqrt{6 l(l+1)} \sqrt{2 l+1} \\
& \times(-1)^{J+l+1 / 2}\left\{\begin{array}{ccc}
S_{B} & S_{B^{\prime}} & 1 \\
l & l & J
\end{array}\right\} \int_{0}^{R} d x x^{2}\left(2 N_{s}{ }^{2} \frac{1}{x} j_{0}\left(\omega_{s} x\right) j_{1}\left(\omega_{s} x\right) j_{l}(k x) j_{l}\left(k^{\prime} x\right)\right) .
\end{aligned}
$$

\begin{tabular}{|c|c|c|c|c|c|c|c|c|}
\hline \multirow{3}{*}{$\bar{I}_{\alpha}$} & \multicolumn{4}{|c|}{$\lambda_{\alpha \beta}^{t, I}$} & \multicolumn{4}{|c|}{$\lambda_{\alpha \beta}^{\text {SO,I I }}$} \\
\hline & & & & & & & & \\
\hline & $\pi N$ & $\pi \Delta$ & $\pi N$ & $\pi \Delta$ & $\pi N$ & $\pi \Delta$ & $\pi N$ & $\pi \Delta$ \\
\hline$\pi N$ & -1 & 0 & $\frac{1}{2}$ & 0 & $-\frac{5}{3}$ & $\frac{-2 \sqrt{2}}{3}$ & $\frac{5}{6}$ & $\frac{-2 \sqrt{5}}{3}$ \\
\hline$\pi \Delta$ & 0 & $-\frac{5}{2}$ & 0 & -1 & $\frac{-2 \sqrt{2}}{3}$ & $\frac{5 \sqrt{10}}{6}$ & $\frac{-2 \sqrt{5}}{3}$ & $\frac{\sqrt{10}}{3}$ \\
\hline
\end{tabular}

TABLE I. The coupling constants for $\pi N$ scattering through the contact term for isospin $I=\frac{1}{2}$ and $I=\frac{3}{2}$ [cf. Eqs. (2.5) and (2.6)]. 
Once again the coupling constants $\lambda_{\alpha \beta}^{\text {so, } I}$ are given in Table I. The curly brackets denote a $6 j$ symbol. It is interesting that the time component, Eq. (2.5), is purely central, whereas the spatial term is purely a spin-orbit force.

The direct and crossed Born terms, shown in Figs. 1(b) and 1(c), respectively, arise through the Yukawa interactionthe next-to-last term in Eq. (2.1). Again evaluating this between MIT bag states for the nucleon and/or $\Delta$, we find that the matrix element is ${ }^{17}$

$$
V_{B M, B_{i}}(\mathbf{k})=\sum_{I, i} \frac{\lambda_{B M, B_{i}}}{2 f} \frac{1}{\left[(2 \pi)^{3} 2 \omega_{M}(k)\right]^{1 / 2}} C_{I_{B} I_{M} I_{I, I_{B}} i_{i} i_{i}{ }^{i}} \sum_{J M}\left[\frac{4 \pi}{3}\right)^{1 / 2} Y_{l m}^{*}(\hat{\mathbf{k}}) C_{S_{B} B^{1 J}}^{s_{B} m M} \delta_{J, S_{B_{i}}} f_{B M, B_{i}}(k),
$$

where

$$
f_{B M, B_{i}}(k)=i\left(\frac{\omega_{s} R}{\omega_{s} R-1}\right) \frac{1}{R} j_{1}(k R) .
$$

(See Table II.) Because the Lagrangian density given in Eq. (2.1) is chiral symmetric, the Goldberger-Treiman relationship is satisfied in this model. In particular, the $\pi N N$ coupling constant in Eqs. (2.7) and (2.8) is directly proportional to the bag-model axial-vector charge of the nucleon. However, the latter is about $13 \%$ lower than experiment. In some papers this discrepancy has been attributed to c.m. corrections ${ }^{22}$ or to renornalization effects. ${ }^{23}$ Whatever the origin of the required enhancement it should be no surprise in Sec. III when we find phenomenologically that such an increase is necessary. Finally, we observe that the shape of the form factor given in Eq. (2.8) is identical to that found in the surface-coupling version of the CBM-a result which is only true when the orbital of the struck quark does not change.

When the baryon formed by pion absorption $\left(B_{i}\right)$ is the Roper resonance [assumed to be a $(1 s)^{2}(2 s)^{1}$ bag state; we are aware of a great deal of speculation about the nature of this state], the form factor becomes ${ }^{17}$

$$
\begin{aligned}
f_{B M, B_{i}}(\mathbf{k})= & 2 R^{2} j_{1}(k r)\left[N_{0} j_{0}\left(\omega_{s} R\right)\right]\left[N_{1} j_{1}\left(\omega_{1} R\right)\right]+\left[\omega_{1}-\omega_{s}-\omega(k)\right] \int_{0}^{R} d x x^{2} j_{1}(k x) \\
& \times N_{0} N_{1}\left[j_{1}\left(\omega_{1} r\right) j_{0}\left(\omega_{s} r\right)-j_{1}\left(\omega_{s} r\right) j_{0}\left(\omega_{1} r\right)\right] .
\end{aligned}
$$

Here $\omega_{1}$ and $N_{1}$ are, respectively, the energy and wave-function normalization constant of the first $s$-wave excited state in the MIT bag model $\left(\omega_{s} R=2.04, \omega_{1} R=5.04\right)$.

The direct Born term $\left[v_{\alpha, \beta}^{d}\right.$ Fig. 1(b)] is obtained by putting two vertices together with an intermediate energy denominator

$$
v_{\alpha \beta}^{d}\left(\mathbf{k}, \mathbf{k}^{\prime}\right)=\frac{v_{B M, B_{i}}(\mathbf{k}) v_{B^{\prime} M^{\prime}, B_{i}}^{*}(\mathbf{k})}{E-M_{B_{i}}} .
$$

It should be noted that the mass of the intermediate baryon appearing here $\left(\boldsymbol{M}_{B_{i}}\right)$ is not the physical mass of the intermediate nucleon, $\Delta$, or Roper. Rather it is a bare mass which gets renormalized by the multiple scattering ${ }^{11}-$ see, e.g., Fig. 2.

The crossed Born graphs illustrated in Fig. 1(c) are more complicated because of the presence of (at least) three intermediate particles. In order to simplify the numerical work we make several approximations in dealing with these graphs. First, the recoil kinetic energy of the intermediate baryon will be neglected. (In practice we retain only those terms where the intermediate baryon is a nucleon or a $\Delta$.) Second, we follow Refs. 11 and 24 in approximating the energy denominator for $B_{i}=N$ as

$$
\frac{1}{E-m_{N}-\omega(k)-\omega\left(k^{\prime}\right)} \simeq-\frac{E-m_{N}}{\omega(k) \omega\left(k^{\prime}\right)} .
$$

With these approximations the crossed Born term contributes only to $p$-wave scattering and yields the potential

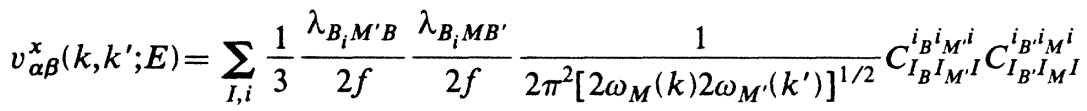

$$
\begin{aligned}
& \times \sum_{J, M, m, m^{\prime}} C_{S_{B} 1 J}^{s_{B^{m}} m M} C_{S_{B^{\prime}} 1 J}^{s_{B^{\prime}} m^{\prime} M} Y_{l m^{\prime}}^{*}\left(\hat{\mathbf{k}}^{\prime}\right) Y_{l m}(\hat{\mathbf{k}})(-1)^{S_{B^{\prime}}+S_{B}+I_{B}+I_{B^{\prime}}+I_{M}+I_{M^{\prime}}} \\
& \times\left(2 S_{B}+1\right)^{1 / 2}\left(2 S_{B^{\prime}}+1\right)^{1 / 2}\left(2 I_{B}+1\right)^{1 / 2}\left(2 I_{B^{\prime}}+1\right)^{1 / 2}\left\{\begin{array}{ccc}
I_{M} & I_{B} & I \\
I_{M^{\prime}} & I_{B^{\prime}} & I_{B_{i}}
\end{array}\right\}\left\{\begin{array}{ccc}
S_{B} & 1 & J \\
S_{B^{\prime}} & 1 & S_{B_{i}}
\end{array}\right\} \\
& \times f_{B_{i} M, B^{\prime}}^{*}(k) \frac{1}{E-m_{B_{i}}-\omega_{M}(k)-\omega_{M^{\prime}}\left(k^{\prime}\right)} f_{B_{i} M^{\prime}, B^{\prime}}\left(k^{\prime}\right) .
\end{aligned}
$$




\section{B. The scattering equation}

To obtain the full scattering amplitude we iterate the Born terms discussed earlier by treating their sum as a potential in the (relativistic) Lippman-Schwinger equation

$$
t_{\alpha \beta}\left(\mathbf{k}, \mathbf{k}^{\prime} ; E\right)=v_{\alpha \beta}\left(\mathbf{k}, \mathbf{k}^{\prime} ; E\right)+\int d^{3} q \sum_{\gamma} v_{\alpha \gamma}(\mathbf{k}, \mathbf{q})\left[E-\left(q^{2}+m_{\gamma}{ }^{2}\right)^{1 / 2}-\left(q^{2}+M_{\gamma}{ }^{2}\right)^{1 / 2}\right]^{-1} t_{\gamma \beta}\left(\mathbf{q}, \mathbf{k}^{\prime} ; E\right)
$$

In this equation $v_{\alpha \beta}$ is the sum of all four processes shown in Fig. 1. We have already shown in Fig. 2 how this procedure generates certain self-energy diagrams. It is also worthwhile to note that almost all vertex renormalization is generated this way too. Figure 3(a) illustrates a few of the graphs generated by solving Eq. (2.14) which renormalize the $N N \pi$ vertex. In Fig. 3(b) we show an example of a contribution which is omitted-because it involves a two-pion intermediate state. The effect of omitting these particular vertex renormalization processes should lie well within the (10-15\%) uncertainty already cited below Eq. (2.8).

\section{Discussion}

One of the hotly debated topics in the field of chiral bag models is the "best" value for the bag radius $R$. This parameter enters our calculations in two ways. First, it controls the form factors at all vertices. As $R$ decreases, these form factors become "harder" and multiple scattering becomes more important. Second, the bag size determines the strength of the spin-orbit term. In the limit where $k, k^{\prime}$ are small all other pieces of the potential are independent of $R$, but the spin-orbit term is directly proportional to it. In contrast with the $\mathrm{KN}$ system, ${ }^{18}$ the spin-orbit term actually plays a small role for pionnucleon scattering.

In concluding this section it is worthwhile to compare the CBM used here with the relativistic potential model of Tegen, Brockmann, and Weise.$^{25}$ The latter has of course only been presented in a pseudoscalar version which is unsuitable for treating $s$ waves. However, by making the same chiral rotation used by Thomas, ${ }^{7}$ that model can be made to look just like the present one. Indeed the contact interaction would look formally the same-only the quark wave functions used would be slightly different (having smooth tails in the potential model). The strength of the time component for small $k$ and $k^{\prime}$ would be identical to ours. For appropriately chosen sizes we would expect these two models to yield very similar results.

TABLE II. The coupling constants for the Yukawa term $\left[\lambda_{B M, B_{i}}\right.$ of Eq. $\left.(2.7)\right]$.

\begin{tabular}{lcc}
\hline \hline$B M$ & $\pi N$ & $\pi \Delta$ \\
$B_{i} \backslash$ & & \\
\hline$N$ & 5 & $+4 \sqrt{2}$ \\
$\Delta$ & $+2 \sqrt{2}$ & 5 \\
$R$ & $\frac{5}{\sqrt{3}}$ & $+4 \sqrt{2}$ \\
\hline
\end{tabular}

\section{RESULTS}

\section{A. Scattering lengths}

In the $s$ wave the time component of the contact interaction and the change of mass term [Eq. (2.2)] are all that contribute. Using $f=93 \mathrm{MeV}$ the Born approximation yields $a_{1}=0.22 \mathrm{fm}$ and $a_{3}=-0.11 \mathrm{fm}$, independent of the bag radius. The same results would be obtained in any chiral quark model which included the contact interaction. We note also that these values are very close to those obtained from $\rho$-meson exchange $(0.24$ and -0.12 $\mathrm{fm}$ in Ref. 26). These results agree very well with the experimental results ${ }^{27}$

$$
a_{1}=0.29 \pm 0.01 \mathrm{fm}, a_{3}=-0.14 \pm 0.01 \mathrm{fm},
$$

or $^{28}$

$$
a_{1}=0.24 \mathrm{fm}, a_{3}=-0.14 \mathrm{fm}
$$

Within the CBM, however, this agreement seems fortuitous. By keeping only the Born term we do not respect unitarity. If we do unitarize by iterating the potential in the relativistic Lippmann-Schwinger equation (2.14) we find $^{21} a_{1}=0.42 \mathrm{fm}, a_{3}=-0.07 \mathrm{fm}$. Thus the multiple scattering, which is unavoidable in our model, generates too much isoscalar attraction. One remedy for this, which was suggested in Ref. 21, is that the pion may ef-

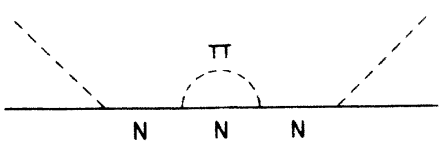

(a)

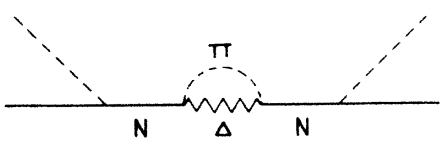

(b)

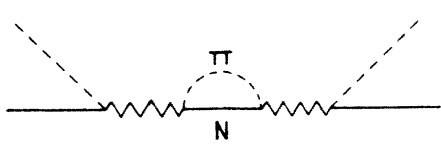

(c)

FIG. 2. Graphs generated by summing the multiple scattering series for the potentials of Fig. 1, which shift the mass of the bare nucleon (a), (b) and delta (c). 


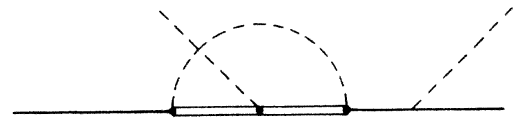

$(N, \Delta)(N, \Delta)$

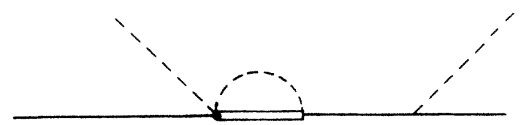

$(N, \Delta)$

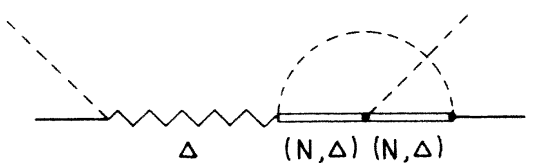

(a)

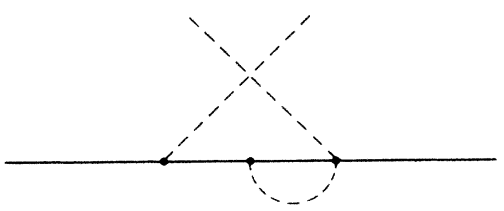

(b)

FIG. 3. (a) Some low-order vertex renormalization graphs included by solving Eq. (2.14) in our model; (b) an example of a (less important) vertex renormalization graph omitted here.

fectively have a larger mass inside the bag than outside.

With a bag radius equal to $1 \mathrm{fm}$ and $f=97 \mathrm{MeV}$ (rather than $93 \mathrm{MeV}$, because we have omitted renormalization effects which would lower the potential strength ${ }^{17}$ ) we find that $\delta m=93 \mathrm{MeV}$ [in Eq. (2.2)] reproduces the experimental data.

The crucial point of this analysis is that the pion-mass change inside the bag is only about $20 \%$. For smaller bag radii the multiple scattering is more important and the value of $\delta m$ required gets larger, e.g., $\delta m=130 \mathrm{MeV}$ for $R=0.8 \mathrm{fm}$. When $R$ gets as small as $0.5 \mathrm{fm}$, the pion mass inside the bag is three times larger than its free value, and $f$ must be set at $117 \mathrm{MeV}$. Even though this is a fairly large effect the pion is still by no means excluded from the bag interior, and $f$ has a value which might easily be justified by renormalization effects. It seems to us that models in which pions are totally excluded from the bag may have difficulty fitting these scattering lengths unless virtual $q \bar{q}$ pair creation is explicitly taken into account.

\section{B. Scattering at finite energy}

From now on we show the results of our calculations with $R=1 \mathrm{fm}$, and fix $f$ and $\delta m$ from the analysis of the scattering lengths. (The results for smaller values of $R$ are qualitatively similar.) With $f, \delta m$, and $R$ fixed the $s$ wave scattering is completely determined. The resulting phase shifts are shown in Figs. 4 and 5 in comparison with the experimental phase shifts of Arndt and Roper. ${ }^{29}$ We see that except near threshold, where we forced agreement on the scattering lengths, the data are not well reproduced. Phenomenologically it seems that we would need additional long-range attraction and short-range

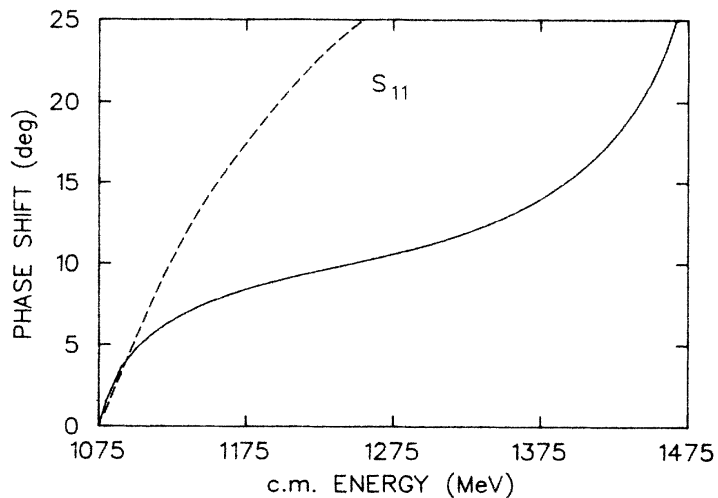

FIG. 4. A comparison between our calculated phase shifts (dashed line), and the data of Ref. 31, for the $S_{11}$ channel.

repulsion to preserve the scattering lengths while improving the phase shifts at higher energy.

At this stage it is worthwhile to compare our $s$-wave results with those of Hirt and co-workers, ${ }^{30}$ who calculated pion-nucleon scattering in the one-boson-exchange (OBE) model. In their work the parameters were chosen to be consistent with the Bonn $N-N$ potential. ${ }^{31}$ They found that the major contributions to $s$-wave scattering came from $\rho$ and $\sigma$ exchange. With $\rho$ exchange alone their results were very close to ours-once again indicating that our contact term has the same physics. When $\sigma$ exchange is added their results are too attractive. Thus they, too, need some extra short-range repulsion (and perhaps reduced strength for the $\sigma N N$ coupling).

While our model contains no $\sigma$ meson, the underlying nonlinear CBM Lagrangian density does contain pionpion scattering terms. These terms, particularly in the $s$ wave, could give an effect much like the $\sigma$ meson. This certainly deserves to be investigated further.

Next we turn to the $p$ waves, where the channel coupling is important and we retain both the pion-nucleon and pion- $\Delta$ channels. In the $P_{13}$ and $P_{31}$ channels-the

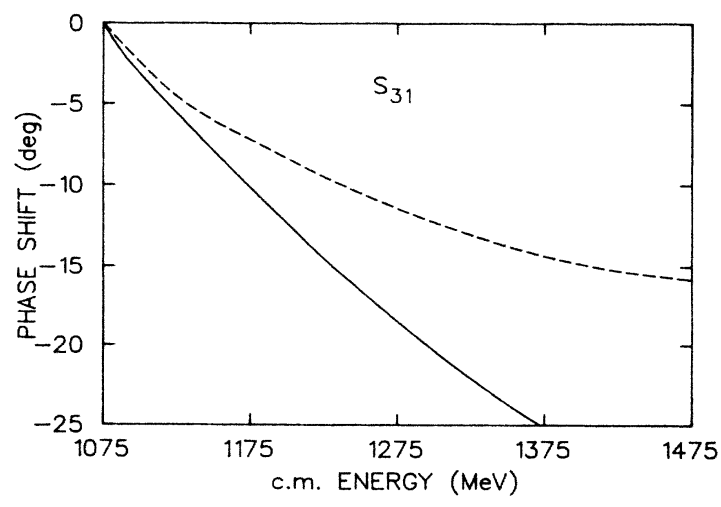

FIG. 5. Same as Fig. 4 for the $S_{31}$ channel. 


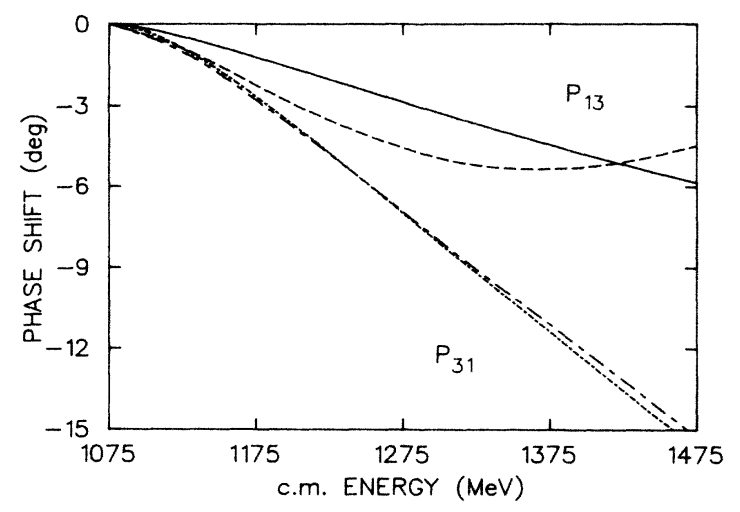

FIG. 6. A comparison of our results (dashed curve and longdashed-short-dashed curve) for the $P_{13}$ and $P_{31}$ phase shifts, with the data of Ref. 31 (solid and small-dashed curves, respectively).

so-called "small $p$ waves"-there are no resonances below $1700 \mathrm{MeV}$. Thus the scattering in these channels should occur mainly through the contact interaction and the crossed Born terms. To be consistent with our calculation in the $P_{33}$ channel (see next paragraph) we have increased the $\pi N N$ coupling by $25 \%$. As we see in Fig. 6 , the agreement between our calculations and the data is quite good. If we use the purely unrenormalized values for the couplings $\left(\delta m=0, f_{\pi}=93 \mathrm{MeV}\right.$, etc.) the results do not change dramatically. In the $P_{31}$ channel the phase shift becomes less negative by $7 \%$ while in the $P_{13}$ channel the minimum value of the phase shift becomes $-3.3^{\circ}$ rather than $-5.3^{\circ}$.

The situation in the $P_{11}$ and $P_{33}$ channels is much more complicated because of the presence of low-lying $s$ channel poles [nucleon $(N)$, delta $(\Delta)$, and Roper resonance $(R)$ ]. We have already described in connection with Fig. 2 (Sec. II A) how multiple scattering renormalizes the masses of these states. Thus we are free to adjust the bare masses $\left(M_{B_{i}}\right)$ to put the physical $\Delta$ and Roper resonances at the correct places. With regard to the coupling constants, we have mentioned that some vertex renormalization is omitted from our calculations. Furthermore, the $\pi N N$ coupling constant implied by Eqs. (2.7) and (2.8) is too small by about $12 \%$. Phenomenologically, we obtain the best fit to the experiment phase shifts with the bare, CBM coupling constants multiplied by a factor of 1.25 (recall $f=97 \mathrm{MeV}$ ), which is well within the uncertainty cited.

With this adjustment of the coupling constants the agreement of the calculated phase shifts with data in the $\boldsymbol{P}_{33}$ channel is excellent-see Fig. 7 , where the data is the solid curve, and the full calculation is the long-dashed curve. For interest we also show the effect of turning off the spin-orbit potential [Eq. (2.7)] arising from the contact interaction (short-dashed curve). This has the effect of reducing the renormalized $\Delta \Delta \pi$ coupling constants, ${ }^{23}$ hence reducing the $\Delta$ self-energy and pushing the $P_{33}$ resonance up in mass. [If the bare mass, which is $1452 \mathrm{MeV}$, was moved down (by $33 \mathrm{MeV}$ ) to give a resonance at the

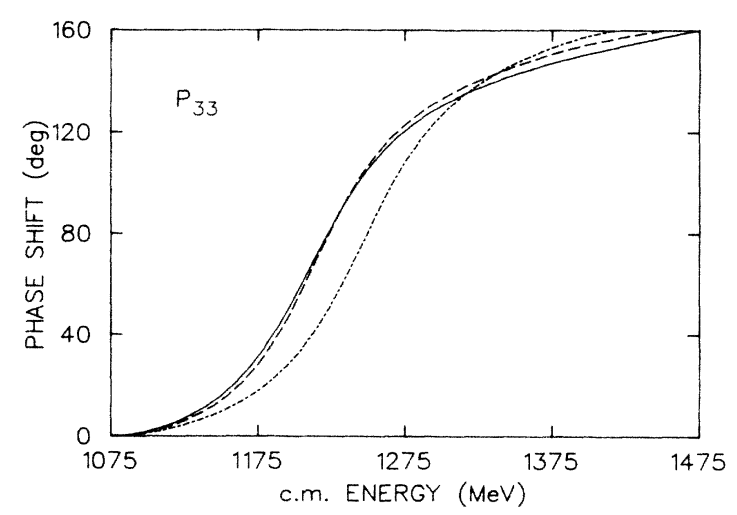

FIG. 7. Same as Fig. 4 for the $P_{33}$ channel. The dotted curve shows the effect of arbitrarily turning off the spin-orbit term, Eq. (2.6).

correct position, the width would be too small.] In Fig. 8 we show the phase shift using the unadjusted couplings $\left(\delta m=0, f_{\pi}=93 \mathrm{MeV}\right.$, etc.). The width is seen to be too narrow. Since at the present level of sophistication the bare mass cannot be reliably calculated it must be adjusted to fit the resonance mass even in this case $\left(M_{\Delta}^{0}=1380\right.$ $\mathrm{MeV})$.

Finally, we show in Fig. 9 the predictions for the (real part of the) $P_{11}$ phase shifts with $N$ and $R$ bare masses equal to 1200 and $1510 \mathrm{MeV}$, respectively. It is clearly not a very satisfactory description of the scattering data in this channel. We have convinced ourselves that it is possible to improve the agreement with the data by arbitrarily varying the $R N \pi$ and $R \Delta \pi$ coupling constants. However, we do not consider it worthwhile to show those results. First, as has often been pointed out, it is probably not correct to think of the Roper as a simple $\left|1 s_{1 / 2}{ }^{2}, 2 s_{1 / 2}\right\rangle$ configuration in a static bag. Second, in this paper we have followed the most practical path in treating baryonmeson scattering over a wide energy range-that is, we

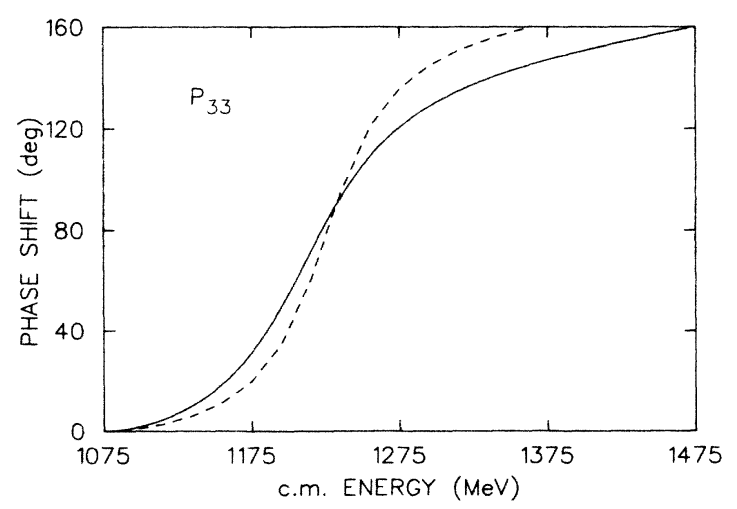

FIG. 8. A comparison of our calculation with the unadjusted input parameters (see text) to the experimental data of Ref. 31 . 


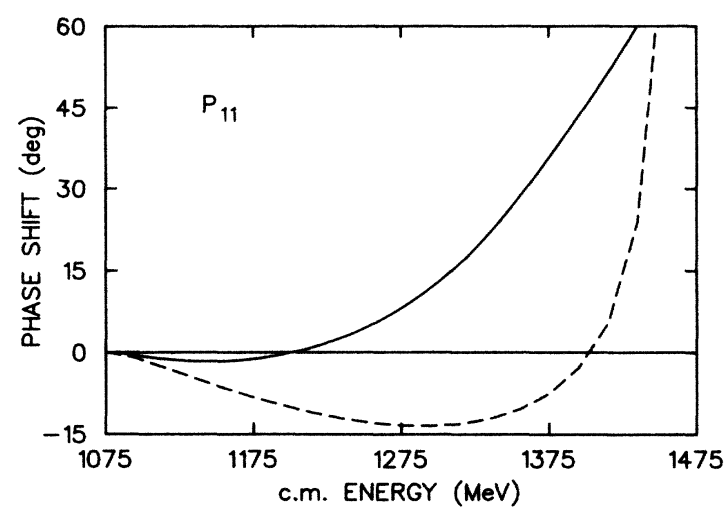

FIG. 9. Same as for Fig. 4 for the $P_{11}$ channel.

have ignored explicit renormalization procedures. On the other hand, for the $P_{11}$ channel one cannot avoid wavefunction renormalization in a consistent treatment. ${ }^{7,11}$ Rather than open this issue here, we prefer to leave a full study of both these aspects of the $P_{11}$ channel for a future, comprehensive study.

We shall not report the results for $d$-wave scattering here. As we have already remarked, the present model does seem to miss some long-range attraction, and $1=2$ is, of course, more sensitive to long-range forces. We expect to report on the higher partial waves after a more careful study of the two-pion contribution.

Before leaving this section we will briefly compare the CBM results with those from the Skyrme-model ${ }^{32,33} \mathrm{cal}$ culations. In the $P_{13}$ and $P_{31}$ partial waves the Skyrmemodel results are quite comparable to the CBM results indicating that phase shifts in both models may be largely due to the chiral symmetry. In the $P_{11}$ channel the two models give equally poor results, although most likely for different reasons. The comparison in the $P_{33}$ channel is more difficult since we do not predict the location of the $\Delta$ resonance but rather fit it. With this adjustable parameter the CBM can do quite well, while the Skyrme model has problems. In the $s$ waves one of the dominant features in the CBM is the contact term which gives an isospin splitting comparable to the experimental splitting. On the other hand, in the Skyrme model this splitting is much more difficult to get. ${ }^{32,33}$

\section{CONCLUSION}

We have made a study of all $s$ - and $p$-wave pionnucleon phase shifts in the volume coupling version of the CBM. The agreement between theory and experiment in the small $p$ waves, and in $P_{33}$, is quite impressive.

With regard to the $P_{33}(1231)$ resonance, our earlier conclusion, ${ }^{11}$ that it is primarily a three-quark bag state, is confirmed. In the $P_{11}$ channel the agreement is far less satisfactory. Rather than enter into the controversy over the nature of the underlying bag state we simply note recent progress in the treatment of surface oscillations which would not be inconsistent with our approach. ${ }^{34}$

In $s$ wave the theoretical situation is not satisfactory, and it seems that our model is probably missing some long-range attraction-possibly associated with uncorrelated two-pion exchange. Nevertheless, to put matters in perspective, it is impressive that such a simple model does reproduce the $S_{11}$ and $S_{31}$ phase shifts within $25 \%$ over an energy interval of $400 \mathrm{MeV}$.

As we discussed in Sec. II C, we believe that little would be changed by using a relativistic potential model (rather than a bag), provided the representation of chiral symmetry was the same. That is, the volume, pseudovector coupling with a contact interaction is essential. We feel that the present work, together with the results for $K N, \bar{K} N$, and pion photoproduction make chiral quark models with pseudoscalar coupling obsolete.

For the future, we believe that even more information can be obtained from pion-nucleon scattering, and further work is in progress. It would be most helpful if other models of hadron structure were also used to calculate meson-baryon phase shifts-especially in nonresonant partial waves.

\section{ACKNOWLEDGMENTS}

It is a pleasure to acknowledge helpful conversations with P. A. M. Guichon, G. A. Miller, and A. S. Rinat. This work was supported in part by research funds from the Australian Research Grants Scheme (Australia), the University of Adelaide, the Natural Sciences and Engineering Research Council (Canada), Conselho Nacional de Pesquisas do Brasil $\left(\mathrm{CNP}_{q}\right)$, and Financiadora de Estudos e Projectos (FINEP) (Brazil).
${ }^{1}$ T. Inoue and T. Maskawa, Prog. Theor. Phys. 54, 1833 (1975).

${ }^{2}$ A. Chodos and C. B. Thorn, Phys. Rev. D 12, 2733 (1975).

${ }^{3}$ G. E. Brown and M. Rho, Phys. Lett. 82B, 177 (1979).

${ }^{4}$ G. A. Miller, S. Théberge, and A. W. Thomas, Phys. Lett. 91B, 192 (1980); Comments Nucl. Part. Phys. 10, 101 (1981).

${ }^{5}$ R. L. Jaffe, in Pointlike Structures Inside and Outside Hadrons, proceedings of the Seventeenth International School of Subnuclear Physics, Erice, 1979, edited by A. Zichichi (Plenum, New York, 1982), p. 99.

${ }^{6}$ G. E. Brown, in Progress in Particle and Nuclear Physics, edited by D. Wilkinson (Pergamon, London, 1982), p. 147.

${ }^{7}$ A. W. Thomas, Adv. Nucl. Phys. 13, 1 (1984).

${ }^{8}$ C. E. De Tar and J. F. Donoghue, Annu. Rev. Nucl. Part. Sci.
33, 235 (1983).

${ }^{9}$ A. W. Thomas, J. Phys. G 7, L283 (1981).

${ }^{10}$ A. Szymacha and S. Tatur, Z. Phys. C 7, 311 (1981).

${ }^{11}$ A. W. Thomas, S. Théberge, and G. A. Miller, Phys. Rev. D 24, 216 (1981); 22, 2838 (1980); 23, 2106(E) (1981).

${ }^{12}$ A. S. Rinat, Nucl. Phys. A377, 341 (1982).

${ }^{13}$ Z. Z. Israilov and M. M. Musakhanov, Phys. Lett. 104B, 173 (1981).

${ }^{14}$ B. K. Jennings and O. V. Maxwell, Nucl. Phys. A422, 589 (1984).

${ }^{15} \mathrm{G}$. Kälbermann and J. Eisenberg, Phys. Rev. D 28, 66 (1983).

${ }^{16} \mathrm{G}$. Kälbermann and J. Eisenberg, Phys. Rev. D 28, 71 (1983).

${ }^{17}$ E. A. Veit, B. K. Jennings, R. C. Barrett, and A. W. Thomas, 
Phys. Lett. 137B, 415 (1984); E. A. Veit, B. K. Jennings, A. W. Thomas, and R. C. Barrett, Phys. Rev. D 31, 1033 (1985).

${ }^{18}$ E. A. Veit, A. W. Thomas, and B. K. Jennings, Phys. Rev. D 31, 2242 (1985).

${ }^{19}$ E. V. Shuryak, CERN Yellow Report No. 83-01 (unpublished).

${ }^{20}$ A. W. Thomas, Prog. Nucl. Part. Phys. 11, 325 (1984).

${ }^{21}$ B. K. Jennings, E. A. Veit, and A. W. Thomas, Phys. Lett. 148B, 338 (1984).

22J. F. Donoghue and K. Johnson, Phys. Rev. D 21, 1975 (1980); C. Carlson and M. Chachkhunasvili, Nordita report, 1981 (unpublished).

${ }^{23}$ G. A. Miller, M. A. Morgan, and A. W. Thomas (unpublished).

${ }^{24}$ G. A. Miller, in Meson-Nuclear Physics-1979, proceedings of the 2nd International Topical Conference, Houston, edited by E. V. Hungerford III (AIP Conf. Proc. No. 54) (AIP, New York, 1979), p. 561.

${ }^{25}$ R. Tegen, R. Brockmann, and W. Weise, Phys. Lett. 125B, 9
(1983).

${ }^{26}$ F. Hackenberg and H. J. Pirner, Ann. Phys. (N.Y.) 112, 401 (1978).

${ }^{27}$ V. S. Zidell, R. N. Arndt, and L. D. Roper, Phys. Rev. D 21, 1289 (1980).

28J. Stahov, Karlsruhe Report No. TKP82-16 (unpublished).

${ }^{29}$ R. A. Arndt and L. D. Roper, scattering analyses interactive dial-in (SAID program) from VPI and SU, PN84-7 data Ford 08/02/84.

${ }^{30}$ D. Hirt, diploma thesis, 1979; W. Ferchländer, D. Hirt, and D. Schütte, Bonn report (unpublished).

${ }^{31}$ R. Machleidt, in Quarks and Nuclear Structure, proceedings of the 3rd Klaus Erkelena Symposium, Bad Honnef, 1983, edited by K. Bleuler (Lecture Notes in Physics, Vol. 197) (Springer, Heidelberg, 1984).

${ }^{32}$ M. P. Mattis and M. Karliner, Phys. Rev. D 31, 2833 (1985).

${ }^{33}$ G. Eckart, A. Hayashi, and G. Holzworth, Siegen report, 1985 (unpublished).

${ }^{34}$ P. A. M. Guichon (unpublished). 